\title{
SUBDIRECTLY IRREDUCIBLE MEMBERS OF PRODUCTS OF LATTICE VARIETIES
}

\author{
GEORGE GRÄTZER AND DAVID KELLY
}

(Communicated by William C. Waterhouse)

\begin{abstract}
In this paper we prove:
THEOREM. Let $\mathbf{V}$ and $\mathbf{W}$ be nontrivial lattice varieties. If $L \in \mathbf{V} \circ \mathbf{W}$, then there is a subdirectly irreducible $S \in \mathrm{V} \circ \mathrm{W}$ containing $L$ as a sublattice. Moreover, if $L$ is finite, $S$ can also be chosen to be finite.
\end{abstract}

1. Introduction. Products of group varieties were investigated in H. Neumann [10]. Products of varieties for arbitrary algebras were introduced in A. I. Mal'cev [9]. We started in [6] to investigate products of lattice varieties.

Let $\mathbf{X}$ and $\mathbf{Y}$ be classes of lattices closed under isomorphism. The product of $\mathbf{X}$ and $\mathbf{Y}, \mathbf{X} \circ \mathbf{Y}$, is the class of all lattices $L$ such that there exists a congruence relation $\Theta$ on $L$ with the following two properties: (i) every congruence class of $\Theta$, as a lattice, is in $\mathbf{X}$; (ii) $L / \Theta$ is in $\mathbf{Y}$.

In this paper we prove:

THEOREM. Let $\mathbf{V}$ and $\mathbf{W}$ be nontrivial lattice varieties. If $L \in \mathbf{V} \circ \mathbf{W}$, then there is a subdirectly irreducible $S \in \mathrm{V} \circ \mathrm{W}$ containing $L$ as a sublattice. Moreover, if $L$ is finite, $S$ can also be chosen to be finite.

This is a very strong property. Previously, $\mathbf{L}$ (the variety of all lattices) was the only nontrivial lattice variety known to have this property.

A class $\mathbf{X}$ of algebras in which every algebra can be embedded into a subdirectly irreducible member of $\mathbf{X}$ is sometimes called "subdirectly complete" or "having sufficiently many subdirectly irreducibles." Thus, the Theorem states that if $\mathbf{V}$ and $\mathbf{W}$ are nontrivial lattice varieties, then $\mathbf{V} \circ \mathbf{W}$ is subdirectly complete $(\mathbf{V} \circ \mathbf{W}$ has sufficiently many subdirectly irreducibles).

The Theorem has several consequences. Henceforth, $\mathbf{D}$ denotes the variety of distributive lattices and $\mathbf{T}$ the trivial variety of one-element lattices.

COROLLARY 1. Let $\mathbf{V}$ and $\mathbf{W}$ be nontrivial lattice varieties. If $\mathbf{Z}=\mathbf{V} \circ \mathbf{W}$ is a variety, then $\mathbf{Z}$ is join irreducible. In particular, $\mathbf{L}$ and $\mathbf{D} \circ \mathbf{D}$ are join irreducible.

This statement about $\mathbf{L}$ can be found in [8].

COROLLARY 2. There are continuumly many join irreducible varieties in the lattice of all lattice varieties.

This was also proved by J. Berman [1] using a different construction.

Received by the editors January 2, 1985 and, in revised form, October 28, 1986.

1980 Mathematics Subject Classification (1985 Revision). Primary 06B20.

This research was supported by the NSERC of Canada. 
In [2], A. Day considered the solutions of $\mathbf{X} \circ \mathbf{X}=\mathbf{X}$ for lattice varieties; he proved that only $\mathbf{L}$ and $\mathbf{T}$ provide solutions. We generalize this in

COROLlaRY 3. For varieties $\mathbf{X}$ and $\mathbf{Y}$ of lattices, the equation

$$
\mathbf{X} \circ \mathbf{Y}=\mathbf{X} \vee \mathbf{Y}
$$

has only the obvious solutions $\{\mathbf{X}, \mathbf{Y}\} \cap\{\mathbf{T}, \mathbf{L}\} \neq \varnothing$.

In [5], we gave an alternative proof of Corollary 3. The proof in [5] also depends on the construction of a subdirectly irreducible lattice.

In $\S 2$, we review the concept of a "complete construction scheme" from [6]. We outline the proof of the Theorem in $\S 3$. We give in $\S 4$ the complete construction scheme for the subdirectly irreducible lattice of the Theorem. The Theorem is proved in $\S 5$, and the corollaries in $\S 6$.

2. Complete construction scheme. In [6], we developed a method for constructing a lattice and a congruence relation on the lattice from a family of complete lattices. We shall construct the subdirectly irreducible lattice of the Theorem in $\S 3$ using this method.

A complete construction scheme, $S$, consists of a lattice $K$ (the frame); a family $L_{a}, a \in K$, of complete lattices (the building blocks); the maps $f_{a b}: L_{a} \rightarrow L_{b}$ (the construction maps) for $a, b \in K, a \leq b$, satisfying for $a, b, c \in K, a \leq b \leq c$,

(C1) $f_{a a}$ is the identity map on $L_{a}$,

(C2) $f_{a b}$ is a complete join-homomorphism,

(C3) $f_{a b} f_{b c}=f_{a c}$.

Given a complete construction scheme, $S$, we define the sum of $S, L$, as follows. $L$ is the disjoint union of the $L_{a}, a \in K$.

We partially order $L$ by the rule:

(P) for $x \in L_{a}$ and $y \in L_{b}, x \leq y$ iff $a \leq b$ and $x f_{a b} \leq y$.

Let $\Theta$ be the equivalence relation on $L$ with blocks $L_{a}, a \in K$.

LEMMA [6, Lemmas 6 and 8]. $L$ is a lattice; the $L_{a}, a \in K$, are sublattices of $L ; \Theta$ is a congruence relation on $L$. For $a, b \in K, a \leq b$, we can represent $f_{a b}$ as $x \rightarrow x \vee 0_{b}$ where $0_{b}$ is the zero of $L_{b}$.

3. Proof outline. Let $L \in \mathrm{V} \circ \mathbf{W}$ by virtue of the congruence relation $\Theta$ (that is, the $\Theta$-classes belong to $\mathbf{V}$ and $L / \Theta \in \mathbf{W}$ ); let $K=L / \Theta$. By the Embedding Theorem in [6], we can assume that the congruence classes of $\Theta$ are complete lattices.

We define a complete construction scheme with frame $K \times C_{3}$ as follows (see Figure 1). On the lowest level (i.e., on $K \times\{0\}$ ), the blocks are the blocks of $\Theta$ with a new zero; the maps are the maps in $L$. On the middle level, each block is a complete Boolean lattice $B$ such that $L$ has a join-embedding $\varphi$ into $B$ preserving all existing complete joins. The maps from the lowest level to the middle level are induced by $\varphi$. On the upper level, we put $C_{2}$.

The sum $S$ of this complete construction scheme is the subdirectly irreducible lattice in $\mathbf{V} \circ \mathbf{W}$ containing $L$.

4. The construction. Let $L$ be a lattice, and let $\Theta$ be a congruence relation on $L$. Let $K=L / \Theta$, and for $a \in K$, let $L_{a}$ denote the corresponding congruence 


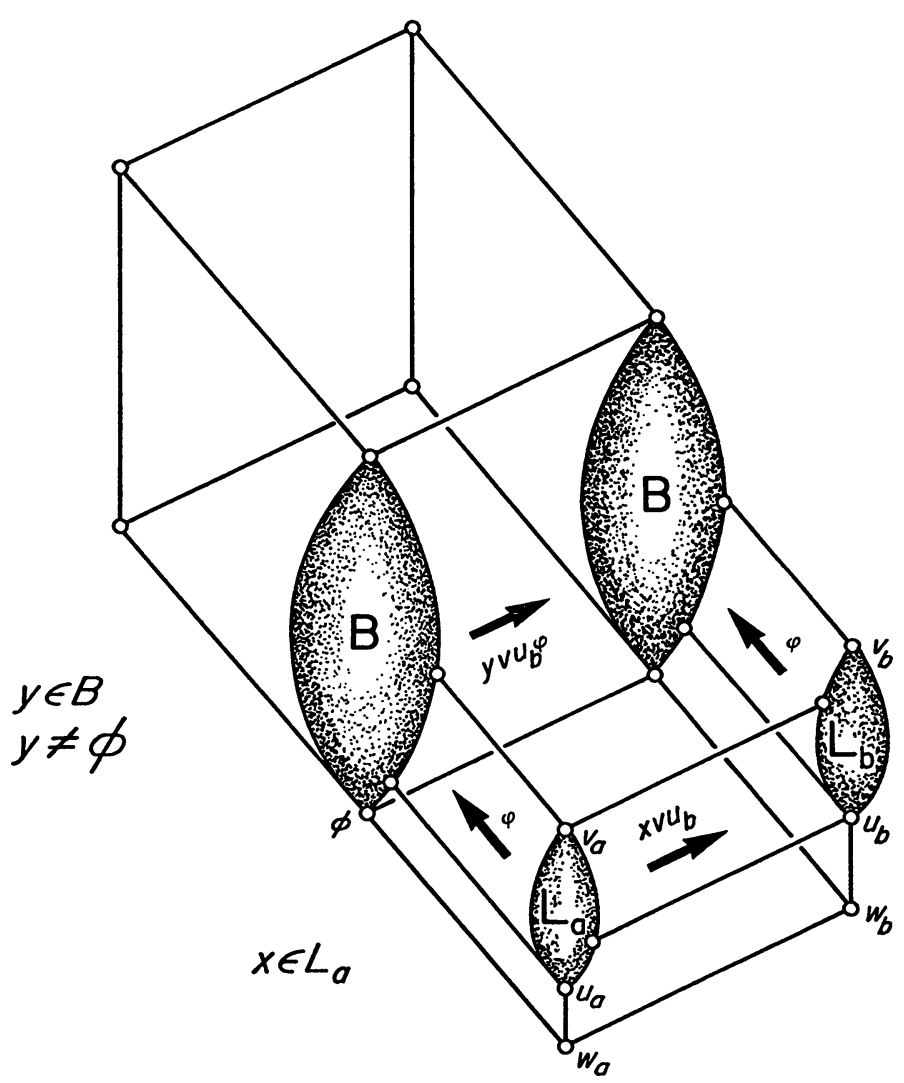

FIGURE 1. Construction details for $a<b$ in $K$

class of $L$. We assume that every $L_{a}, a \in K$, is complete; in particular, $L_{a}$ is an interval of $L, L_{a}=\left[u_{a}, v_{a}\right]$.

Let $B$ be the complete Boolean lattice of all subsets of $L \cup\{\gamma, \delta\}(\gamma, \delta \notin L$, $\gamma \neq \delta)$. We define a map $\varphi: L \rightarrow B$ by

$$
x \varphi=(L-[x)) \cup\{\gamma, \delta\} .
$$

It is well known (and trivial) that $\varphi$ preserves all existing nonempty finite and infinite joins in $L$.

Let $C_{3}=\{0,1,2\}$ be the 3-element chain. For every $\langle a, i\rangle \in K \times C_{3}$, we define a lattice $S_{\langle a, i\rangle}$ as follows:

(S0) $S_{\langle a, 0\rangle}$ is the lattice $L_{a}$ with the new zero $w_{a}$.

(S1) $S_{\langle a, 1\rangle}$ is a Boolean lattice $B \times\{a\}$.

(S2) $S_{\langle a, 2\rangle}$ is $C_{2} \times\{a\}$, where $C_{2}$ is the two-element chain $\{0,1\}$.

Next, we define some of the construction maps. First, for $a, b \in K, a<b$, we define three maps (recall that $u_{b}$ is the zero of $L_{b}$ ).

$f_{\langle a, 0\rangle,\langle b, 0\rangle}$ maps $S_{\langle a, 0\rangle}$ into $S_{\langle b, 0\rangle}$ :

$$
x f_{\langle a, 0\rangle,\langle b, 0\rangle}= \begin{cases}w_{b} & \text { if } x=w_{a}, \\ x \vee u_{b} & \text { if } x \neq w_{a} ; x \vee u_{b} \text { is formed in } L .\end{cases}
$$


$f_{\langle a, 1\rangle,\langle b, 1\rangle}$ maps $S_{\langle a, 1\rangle}$ into $S_{\langle b, 1\rangle}$. Let $x \in S_{\langle a, 1\rangle}$, that is, $x=\langle X, a\rangle$, where $X \subseteq L \cup\{\delta\}$; define

$$
x f_{\langle a, 1\rangle,\langle b, 1\rangle}= \begin{cases}\langle\varnothing, b\rangle & \text { if } x=\langle\varnothing, a\rangle, \\ \left\langle X \cup u_{b} \varphi, b\right\rangle & \text { otherwise. }\end{cases}
$$

This definition makes sense since $u_{b} \in L$, therefore, $u_{b} \varphi \in B$.

$f_{\langle a, 2\rangle,\langle b, 2\rangle}$ is the unique isomorphism between $S_{\langle a, 2\rangle}$ and $S_{\langle b, 2\rangle}$ :

$$
\langle x, a\rangle f_{\langle a, 2\rangle,\langle b, 2\rangle}=\langle x, b\rangle \text { for } x \in C_{2} .
$$

Now for an $a \in K$ we define two maps:

$f_{\langle a, 0\rangle,\langle a, 1\rangle}$ maps $S_{\langle a, 0\rangle}$ into $S_{\langle a, 1\rangle}$ :

$$
x f_{\langle a, 0\rangle,\langle a, 1\rangle}= \begin{cases}\langle\varnothing, a\rangle & \text { if } x=w_{a}, \\ \langle x \varphi, a\rangle & \text { if } x \in L_{a} .\end{cases}
$$

$f_{\langle a, 1\rangle,\langle a, 2\rangle}$ maps $S_{\langle a, 1\rangle}$ into $S_{\langle a, 2\rangle}$ :

$$
x f_{\langle a, 1\rangle,\langle a, 2\rangle}= \begin{cases}\langle 0, a\rangle & \text { if } x=\langle\varnothing, a\rangle, \\ \langle 1, a\rangle & \text { otherwise. }\end{cases}
$$

Finally, we define $f_{\langle a, i\rangle,\langle a, i\rangle}$ as the identity map on $S_{\langle a, i\rangle}$.

Observe that all the maps we have defined map zero, and only the zero, to zero.

Now we verify that from the $S_{\langle a, i\rangle}$ and the maps defined above we can put together a complete construction scheme. First, the building blocks must be complete.

Claim 1. For $\langle a, i\rangle \in K \times C_{3}, S_{\langle a, i\rangle}$ is a complete lattice.

PROOF. This holds by assumption for $i=0$; it is trivial for $i=1$ and 2 .

Next we verify that the maps we have defined satisfy (C2).

ClaIM 2. All the maps $f_{\langle a, i\rangle,\langle b, j\rangle}$ defined in (F0)-(F4) are complete join homomorphisms.

Proof. For (F0), (F1), (F2), and (F4) this is trivial. To verify it for (F3), we only have to observe that $\varphi: L \rightarrow B$ preserves all existing nonempty joins. Since $L_{a}$ is a complete sublattice of $L, \varphi$ restricted to $L_{a}$ preserves all the nonempty complete joins in $L_{a}$, verifying the claim.

The remaining construction maps are obtained by composing the maps that we have already defined. The next claim shows that some compositions of these maps are equal.

ClaIM 3. If $a, b \in K, a<b$, then

(3.1) $f_{\langle a, 0\rangle,\langle b, 0\rangle} f_{\langle b, 0\rangle,\langle b, 1\rangle}=f_{\langle a, 0\rangle,\langle a, 1\rangle} f_{\langle a, 1\rangle,\langle b, 1\rangle}$,

(3.2) $f_{\langle a, 1\rangle,\langle b, 1\rangle} f_{\langle b, 1\rangle,\langle b, 2\rangle}=f_{\langle a, 1\rangle,\langle a, 2\rangle} f_{\langle a, 2\rangle,\langle b, 2\rangle}$.

PROOF. Let $x \in S_{\langle a, 0\rangle}$. If $x=w_{a}$, then both sides of (3.1) map $w_{a}$ into $\langle\varnothing, b\rangle$. If $x \neq w_{a}$, then $x \in L_{a}$. Therefore, using that $\varphi$ preserves finite joins,

$$
\begin{aligned}
x f_{\langle a, 0\rangle,\langle b, 0\rangle} f_{\langle b, 0\rangle,\langle b, 1\rangle} & =\left(x \vee u_{b}\right) f_{\langle b, 0\rangle,\langle b, 1\rangle} \quad(\text { by }(\mathrm{F} 0)) \\
& =\left\langle\left(x \vee u_{b}\right) \varphi, b\right\rangle=\left\langle x \varphi \cup u_{b} \varphi, b\right\rangle \quad(\text { by }(\mathrm{F} 1))
\end{aligned}
$$

while

$$
\begin{aligned}
x f_{\langle a, 0\rangle,\langle a, 1\rangle} f_{\langle a, 1\rangle,\langle b, 1\rangle} & =\langle x \varphi, a\rangle f_{\langle a, 1\rangle,\langle b, 1\rangle} \quad(\text { by }(\mathrm{F} 3)) \\
& =\left\langle x \varphi \cup u_{b} \varphi, b\right\rangle \quad(\text { by }(\mathrm{F} 1))
\end{aligned}
$$

verifying (3.1). Formula (3.2) is trivial by (F1) and (F2). 
Now for any $\langle a, i\rangle\left\langle\langle b, j\rangle\left(a, b \in K, i, j \in C_{3}, a<b, i<j\right)\right.$ we can define a map $f_{\langle a, i\rangle,\langle b, j\rangle}$ :

$$
\begin{aligned}
& f_{\langle a, 0\rangle,\langle b, 1\rangle}=f_{\langle a, 0\rangle,\langle b, 0\rangle} f_{\langle b, 0\rangle,\langle b, 1\rangle}, \\
& f_{\langle a, 0\rangle,\langle a, 2\rangle}=f_{\langle a, 0\rangle,\langle a, 1\rangle} f_{\langle a, 1\rangle,\langle a, 2\rangle}, \\
& f_{\langle a, 0\rangle,\langle b, 2\rangle}=f_{\langle a, 0\rangle,\langle a, 2\rangle} f_{\langle a, 2\rangle,\langle b, 2\rangle} .
\end{aligned}
$$

Now it is easily seen that these maps satisfy (C3).

ClaIM 4. If $\langle a, i\rangle \leq\langle b, j\rangle \leq\langle c, k\rangle$ in $K \times C_{3}$, then

$$
f_{\langle a, i\rangle,\langle b, j\rangle} f_{\langle b, j\rangle,\langle c, k\rangle}=f_{\langle a, i\rangle,\langle c, k\rangle} \text {. }
$$

PROOF. This statement is clear from Claim 3.

We can summarize the results of this section as follows: the complete lattices $S_{\langle a, i\rangle}\left(a \in K, i \in C_{3}\right)$ with the maps $f_{\langle a, i\rangle,\langle b, j\rangle}(\langle a, i\rangle \leq\langle b, j\rangle)$ form a complete construction scheme.

5. Proof of the Theorem. We shall actually prove a slightly more general version of the main result.

THEOREM. Let $\mathbf{V}$ be a nontrivial variety of lattices, and let $\mathbf{W}$ be a class of lattices closed under finite direct products and containing $C_{3}$. Then $\mathrm{V} \circ \mathrm{W}$ is subdirectly complete.

Proof. By the Embedding Theorem ( $\$ 2$ of [6]), every member of $\mathbf{V} \circ \mathbf{W}$ can be embedded into a lattice $L$ with a congruence relation $\Theta$ satisfying (a) every congruence class of $\Theta$ is a complete lattice in $\mathbf{V}$, and (b) $L / \Theta \in \mathbf{W}$. Now we define $K=L / \Theta$ and $L_{a}, a \in K$, as in the previous section and construct the lattices $S_{\langle a, i\rangle},\langle a, i\rangle \in K \times C_{3}, S$ (the sum of the complete construction scheme), and the congruence relation $\Phi$ on $S$. The next two claims verify that $S \in \mathbf{V} \circ \mathbf{W}$.

Claim 5. $S_{\langle a, i\rangle} \in \mathrm{V}$ for $\langle a, i\rangle \in K \times C_{3}$.

PROOF. $S_{\langle a, 0\rangle}$ is $L_{a}$ with a new zero. Since $L_{a} \in \mathbf{V}$ and $\mathbf{V}$ is a variety, $S_{\langle a, 0\rangle} \in \mathbf{V}$. V.

$S_{\langle a, 1\rangle}$ and $S_{\langle a, 2\rangle}$ are Boolean lattices. Since V is nontrivial, $S_{\langle a, 1\rangle}$ and $S_{\langle a, 2\rangle} \in$

Claim 6. $S / \Phi \in \mathbf{W}$.

ProOF. Obviously, $S / \Phi=K \times C_{3}$. By assumption, $K \in \mathbf{W}$. By the hypotheses on $\mathbf{W}, C_{3} \in W$, and $K \times C_{3} \in \mathbf{W}$.

The proof of the Theorem is completed by verifying the following

ClaIM 7. $S$ is subdirectly irreducible.

ProOF. We verify that if $x, y \in S, x<y$, then $\Theta(x, y) \geq \Phi$. This proves that $S$ is subdirectly irreducible.

CASE 1. $x, y \in S_{\langle a, 2\rangle}$. Obviously, $\Theta(x, y)=\Phi$.

CASE 2. $x, y \in S_{\langle a, 1\rangle}$. Since $S_{\langle a, 1\rangle}$ is Boolean, $\Theta(x, y)=\Theta(\langle\varnothing, a\rangle, z)$ for some $z>\langle\varnothing, a\rangle$ (recall that $\langle\varnothing, a\rangle$ is the zero of $S_{\langle a, 1\rangle}$ ).

$$
\langle 0, a\rangle=\langle 0, a\rangle \vee\langle\varnothing, a\rangle \equiv\langle 0, a\rangle \vee z=\langle 1, a\rangle(\Theta(x, y))
$$

and so by Case $1, \Theta(x, y) \geq \Phi$.

CASE 3. $x, y \in S_{\langle a, 0\rangle}$. Joining both sides of the congruence $x \equiv y(\Theta(x, y))$ with $\langle\varnothing, a\rangle$ we get $\langle x \varphi, a\rangle \equiv\langle y \varphi, a\rangle(\Theta(x, y))$ or $\langle\varnothing, a\rangle \equiv\langle y \varphi, a\rangle(\Theta(x, y))$ if $x=w_{a}$. In either case, by Case 2 , we conclude that $\Theta(x, y) \geq \Phi$. 
CASE 4. $x \in S_{\langle a, 0\rangle}$ and $y \in S_{\langle a, 1\rangle}$. Joining with $v_{a}$, we get $v_{a} \equiv v(\Theta(x, y))$, where $v \geq\left\langle v_{a} \varphi, a\right\rangle$. Therefore, $\langle\{\delta\}, a\rangle \equiv\langle\{\delta\}, a\rangle \wedge v_{a}=w_{a}(\Theta(x, y))$, and so $\langle\varnothing, a\rangle \equiv\langle\{\delta\}, a\rangle(\Theta(x, y))$. We conclude, by Case 2 , that $\Theta(x, y) \geq \Phi$.

CASE 5. $x \in S_{\langle a, 1\rangle}$ and $y \in S_{\langle a, 2\rangle}$. Let $p$ and $q$ be distinct atoms of $S_{\langle a, 1\rangle}$. First meeting both sides with $\langle 0, a\rangle$, then joining them with $p$ and $q$ respectively, we obtain from $x \equiv y(\Theta(x, y))$ the congruences $p \equiv\langle 1, a\rangle(\Theta(x, y))$ and $q \equiv\langle 1, a\rangle(\Theta(x, y))$. Hence $\langle\varnothing, a\rangle=p \wedge q \equiv\langle 1, a\rangle(\Theta(x, y))$; this implies that $p \equiv q(\Theta(x, y))$, and a reference to Case 2 concludes this case.

Now let $a, b \in K, a<b$.

CASE 6. $x \in S_{\langle a, i\rangle}$ and $y \in S_{\langle b, i\rangle}$. Let $z_{a, i}$ (resp. $z_{b, i}$ ) be the zero of $S_{\langle a, i\rangle}$ (resp. $\left.S_{\langle b, i\rangle}\right)$. Then meet $x \equiv y(\Theta(x, y))$ with $z_{b, i}$ to obtain $z_{a, i} \equiv z_{b, i}(\Theta(x, y))$. Obviously, this holds for $i=0,1,2$. Join this for $i=1$ with $\langle L \cup\{\gamma, \delta\}, a\rangle$, the unit element of $S_{\langle a, 1\rangle}$; we obtain $\langle L \cup\{\gamma, \delta\}, a\rangle \equiv\langle L \cup\{\gamma, \delta\}, b\rangle(\Theta(x, y))$; now meet with $\langle\{\delta\}, b\rangle: z_{a, 1} \equiv\langle\{\delta\}, b\rangle(\Theta(x, y))$. Case 2 completes the proof.

Finally, observe that if $x<y$, then there must be elements $x_{1}, y_{1}$ satisfying $x \leq x_{1}<y_{1} \leq y$ and one of Case 1 to Case 6. Therefore, $\Theta(x, y) \geq \Phi$.

This completes the proof of the Theorem.

6. Proof of the corollaries. Let $\mathbf{Z}, \mathbf{V}$, and $\mathbf{W}$ be given as in Corollary 1 . Let $K$ be the free lattice on $\aleph_{0}$ generators over $\mathbf{Z}$. By the Theorem, $K$ can be embedded into a subdirectly irreducible lattice $L$ of $\mathbf{Z}$. Obviously, $L$ generates $\mathbf{Z}$. By Jónsson's Lemma (see [8 or $\mathbf{3}]$ ), $\mathbf{Z}$ is join irreducible.

Corollary 2 is immediate from Corollary 1 by a result of [7]; there are continuumly many modular lattice varieties $\mathbf{M}_{i}$, such that all the $\mathbf{M}_{i} \circ \mathbf{D}$ are varieties; all the $\mathbf{M}_{i} \circ \mathbf{D}$ are distinct.

Now we prove Corollary 3 . Let $\mathbf{X}$ and $\mathbf{Y}$ be lattice varieties such that $\mathbf{X}, \mathbf{Y} \notin$ $\{\mathbf{L}, \mathbf{T}\}$. If $\mathbf{X}=\mathbf{Y}$, Corollary 3 is Day's result (see [2]).

If $\mathbf{X} \subset \mathbf{Y}$, then $\mathbf{X} \circ \mathbf{Y}=\mathbf{Y}$. Since $\mathbf{X}$ and $\mathbf{Y}$ are nontrivial, $\mathbf{X} \supseteq \mathbf{D}$. Therefore,

$$
\mathbf{D} \circ \mathbf{D} \subseteq \mathbf{Y} \text {. }
$$

As in [6], let us define $\mathbf{D}^{1}=\mathbf{D}, \mathbf{D}^{n+1}=\mathbf{D}^{n} \circ \mathbf{D}$. It follows from Lemma 3 of [6] that

$$
\mathrm{D}^{n+1} \subseteq \mathrm{D} \circ \mathrm{D}^{n}
$$

hence an easy induction shows that $\mathbf{D}^{n} \subseteq \mathbf{Y}$ for all $n \geq 1$. Again, by A. Day [2], $\mathbf{Y}=\mathbf{L}$, a contradiction.

If $\mathbf{X} \supset \mathbf{Y}$, then $\mathbf{X} \circ \mathbf{Y}=\mathbf{X}$, hence $\mathbf{X} \circ \mathbf{D} \subseteq \mathbf{X}$. A similar argument gives $\mathbf{X}=\mathbf{L}$.

Finally, if $\mathbf{X}$ and $\mathbf{Y}$ are incomparable, then we conclude that $\mathbf{X} \circ \mathbf{Y}$ is a join reducible variety, contradicting Corollary 1.

\section{REFERENCES}

1. J. Berman, Interval lattices and the amalgamation property, Algebra Universalis 12 (1981), 360-375.

2. A. Day, Idempotents in the groupoid of all SP classes of lattices, Canad. Math. Bull. 21 (1978), 499-501.

3. G. Grätzer, General lattice theory, Pure and Appl. Math. Series, Academic Press, New York; Mathematische Reihe, Band 52, Birkhäuser Verlag, Basel; Akademie Verlag, Berlin, 1978. (Russian translation: "MIR" , Moscow, 1982.)

4. __ Universal algebra, 2nd ed., Springer-Verlag, New York, Heidelberg and Berlin, 1979. 
5. G. Grätzer and D. Kelly, On a special type of subdirectly irreducible lattice with an application to products of varieties, C. R. Math. Rep. Acad. Sci. Canada 2 (1980), 43-48.

6. __ Products of lattice varieties, Algebra Universalis 21 (1985), 33-45.

7. _ The lattice variety $\mathbf{D} \circ \mathbf{D}$, Acta. Sci. Math. (Szeged) (to appear).

8. B. Jonsson, Algebras whose congruence lattices are distributive, Math. Scand. 21 (1967), 110121.

9. A. I. Mal'cev, Multiplication of classes of algebraic systems, Siberian Math. J. 8 (1967), 254267; English transl., The metamathematics of algebraic systems, North-Holland, Amsterdam, 1971, pp. 422-446.

10. H. Neumann, Varieties of groups, Ergeb. Math. Grenzgeb., Band 37, Springer-Verlag, New York, 1967.

Department of MAthematics, University of Manitoba, WinNipeg, Manitoba, R3T 2N2 CANADA 